\title{
DWT Based-Video Compression Using (4SS) Matching Algorithm
}

\author{
Marwa Kamel Hussien \\ Assist. Lecturer \\ Lava_85K@yahoo.com \\ Department of Computer Science, College of Science, University of Basrah, Basrah, IRAQ. \\ Dr. Hameed Abdul-Kareem Younis \\ Assist. Professor \\ Hameedalkinani2004@yahoo.com
}

\begin{abstract}
Currently, multimedia technology is widely used. Using the video encoding compression technology can save storage space, and also can improve the transmission efficiency of network communications. In video compression methods, the first frame of video is independently compressed as a still image, this is called intra coded frame. The remaining successive frames are compressed by estimating the disparity between two adjacent frames, which is called inter coded frame. In this paper, Discrete Wavelet Transform (DWT) is used powerful tool in video compression. Our coder achieves a good trade-off between compression ratio and quality of the reconstructed video.
\end{abstract}

The motion estimation and compensation, which is an essential part in the compression, is based on segment movements. The disparity between each two frames was estimated by Four Step Search (4SS) Algorithm. The result of the Motion Vector (MV) was encoded into a bit stream by Huffman encoding while the remaining part is compressed like the compression was used in intra frame. Experimental results showed good results in terms of Peak Signal-to-Noise Ratio (PSNR), Compression Ratio (CR), and processing time.

Keywords: Video Compression, DWT, Motion Estimation, Motion Compensation, Block Matching Algorithm, Four Step Search.

\section{INTRODUCTION}

an images sequence (or video) can be acquired by video or motion picture cameras, or generated by sequentially ordering two-dimension (2d) still images as in computer graphics and animation.

video processing is special cases of digital processing in which signals are processed are video files or video streams. it is extensively used in television sets, digital versatile disks (dvd), video players, ..., etc. although digital video signals can be transferred over the long distances with a low probability of bit error rate, the raw material of digital video requires high bandwidth for transmission and high storage capacities when compared to its analog equivalent. therefore, compression basically is necessary to reduce data, a digitized analog video sequence can comprise of up to $165 \mathrm{mbps}$ of data. to reduce the media overheads for distributing these sequences, the following techniques are commonly employed to achieve desirable reductions in image data [1]:

i. Reduce color nuances within the image

ii. Reduce the resolution with respect to the prevailing light intensity.

iii. Remove spatial redundancy or correlation between neighboring pixels values.

iv. Compare adjacent images and removes details that are unchanged between adjacent frames in sequence of images.

The first three of above points are image based compression techniques that is called intra frame, where only one frame is evaluated and compressed at a time. The last one is called inter frame, where different adjacent frames are compared as a way to further reduced image data. All of these techniques are based on the term of motion. Motion is an essential aspect of video sequences. The ability to estimate, analyzes, and compensate for relative motion is a common requirement of many video processing, analysis and compression algorithms and techniques. Fig.1 shows flowchart of video compression.

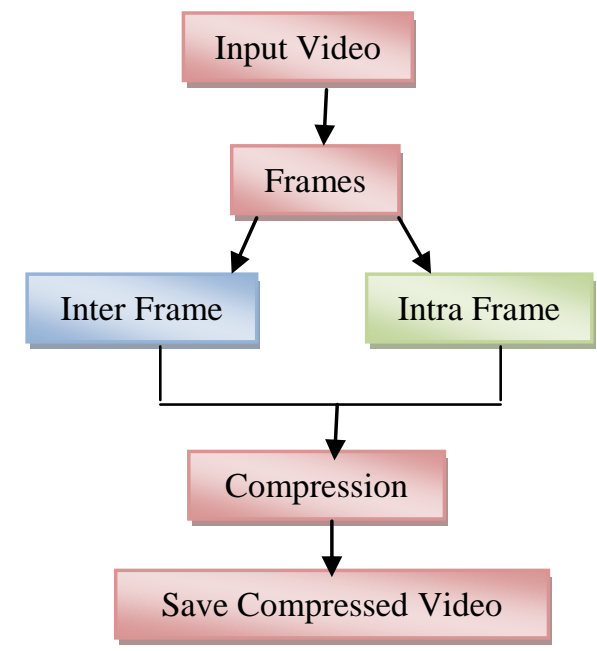

Fig. 1: Flowchart of video compression 
Hassan B. and Malik K. [2] have proposed a method for video compression based on skipping some frames which have little information from the fames sequence. They have designed an algorithm to compare two frames on sub frame level and decides which one is more important for the overall video quality as perceived by eye of the user and skipping the frame which have little information.

Bjorn B. [3] Developed a video codec. This codec consist of three parts, transformation, quantization and encoding. These three fundamental parts are used for the purpose of compressing the data. Through the transform, the energy in a picture is concentrated to a small region. These regions are then rounded off through quantization to compress the data. Author has also proposed a method to cope the problem of error that can be introduced through transmitted it over channels by sent side information over another channel to describes the information in yet another way. Consequently, a coding scheme called Multiple has been presented.

Bernabé G et al. [4] present a lossy compression scheme based on the application of the 3D fast wavelet transform to code medical video. This type of video has special features, such as its representation in gray scale, its very few inter frame variations, and the quality requirements of the reconstructed images. These characteristics as well as the social impact of the desired applications demand a design and implementation

of coding schemes especially oriented to exploit them.

Nema M. et al. [5] present wavelet based video compression algorithms. The motion estimation and compensation, which is an essential part in the compression, is based on segment movements. The proposed work based on wavelet transform algorithm like Set Partitioning In Hierarchical Trees (SPIHT) and Stationary Wavelet Transform (SWT).

The work aims to propose an efficient technique for video compression by wavelet transform by using two systems, intra and inter coded frames. In this present work, video compression system is developed using wavelet transform, and 4SS. The rest of this paper is organized as follows. Some of basic principles has been explained in Section 2. We show our proposed video compression system in Section 3. Section 4 give the experimental results. Finally, the paper has been concluded in Section 5.

\section{BASIC PRINCIPLES}

\section{A. Intra and Inter Coded Frames}

The motion estimation and the motion compensation blocks work, only if there is a past frame that is stored. So, question is how do we encode the first frame in a video sequence, for which there is no past frame reference? The answer to this question is fairly straight forward. We treat the first frame of a video sequence like a still image, where only the spatial, i.e., the intra frame redundancy can be exploited [6].

The frames that use only intra frame redundancy for coding are referred to as the intra coded frame. The first frame of every video sequence is always an intra-coded frame. From the second frame onwards, both temporal as well as spatial redundancy can be exploited. Since these frames use inter frame redundancy for data compression, these are referred to as inter coded frame. However, it is wring to think that only the first frame of a video sequence would be intra-coded and the rest inter-coded. In some of the multimedia standards, intra-coded frames are periodically introduced at regular intervals to prevent accumulation of prediction error over frames. It is obvious that intra-coded frames would require more bits to encode as compared to inter-coded frames since the temporal redundancy is not exploited in the form.

\section{B. Discrete Wavelet Transform (DWT)}

The basic operation in wavelet transform is to filter an image with a low pass filter (L) and a high pass filter $(\mathrm{H})$ and down-sample the output by a factor of 2. Both operations on the $\mathrm{x}$ direction, two new images are obtained L and H. They are filtered and down sampled again but this time in the $\mathrm{y}$ direction. Four sub bands images are obtained which can be combined to recover the original one. The same amount of information is present, but this new configuration is more suitable for efficient coding [7].

The inverse wavelet transform is performed by enlarging the wavelet transform data to it is original size. Insert zeros between each of four sub images, and sum the results to obtain the original image [8]. The Haar wavelet is one of the most common used wavelets. It resembles a stepfunction and is defined by:

$$
\begin{aligned}
\text { Lowpass } & =\frac{1}{\sqrt{2}}\left[\begin{array}{ll}
1 & 1
\end{array}\right] \\
\text { Highpass } & =\frac{1}{\sqrt{2}}\left[\begin{array}{ll}
1 & -1
\end{array}\right]
\end{aligned}
$$

\section{C. $\quad$ The Embedded Zero Tree Wavelet (EZW) Quantization}

An EZW encoder was especially designed by Shapiro to be used with wavelet transform. In fact, EZW coding is a quantization method. The EZW encoder is based on progressive encoding to compress an image into a bit stream with increasing accuracy. This means that when more bits are added to the stream, the decoded image will contain more detail. A zero tree is a tree of which all nodes are equal to or smaller than the root. The tree is coded with a single symbol and reconstructed by the decoder as a tree filled with zeroes [8]. 


\section{Arithmetic Encoding}

Arithmetic encoding, and its derivative technique, Qcoding, is used to overcome some of the limitations of Huffman codes. It is a non-block code, in that a single codeword is used to represent an entire sequence of input symbols, in contrast to Huffman coding where a source symbol block corresponds to a codeword block. Instead, it uses the real numbers to represent a sequence of symbols by recursively subdividing the interval between 0 and 1 to specify each successive symbol. The limitation of this technique is the precision required in performing the calculations and arriving at the code word which will represent the entire sequence correctly [9].

\section{E. Huffman Encoding}

A Huffman encoding developed by D.A. Huffman, a Huffman encoder takes a block of input characters with fixed length and produces a block of output bits of variable length. It is a fixed-to-variable length code. Huffman encoding uses a variable length code for each of the elements within the information. This normally involves analyzing the information to determine the probability of elements within the information. The most probable elements are coded with a few bits and the least probable coded with a greater number of bits [10].

\section{F. PSNR and CR}

Evaluation criteria that usually used in digital image and video compression are in two directions. First direction is to evaluate quality of the reconstructed image. Second direction is Compression Ratio (CR). In terms of quality evaluation, two mathematical metrics are used. First one is Mean Square Error (MSE), which measures the cumulative square error between the original and the reconstructed image. Second meter is Peak Signal-to-Noise Ratio (PSNR). The formula for MSE is giving as [11]:

PSNR is the standard method for quantitatively comparing a compressed image with the original. For an 8-bit grayscale image, the peak signal value is 255 . Hence, the PSNR of an $\mathrm{M} \times \mathrm{N}$ 8-bit grayscale image $\mathrm{C}$ and its reconstruction $\mathrm{R}$ is calculated as [11]:

$$
P S N R=10 \log _{10} \frac{255^{2}}{M S E}
$$

where the MSE is defined as:

$$
M S E=\frac{1}{N^{2}} \sum_{i=0}^{N-1} \sum_{j=0}^{N-1}\left[C_{i j}(m, n)-R_{i j}(m, n)\right]^{2}
$$

PSNR is measured in decibels $(\mathrm{dB}), \mathrm{M}$ : height of the image, $\mathrm{N}$ : width of the image.

The second direction of comparing the compressed and the original images is the compression ratio. It is defined as [12]:

$$
\text { Compression Ratio }=\frac{\text { Compressed File Size }}{\text { Uncompressed File Size }}=\frac{\text { Size }_{c}}{\operatorname{Size}_{u}}
$$

In addition to measuring the quality of image, we also measure the compression ratio. Compression ratio is the ratio of the compressed file size to the original file size. In general, the higher the compression ratio, the smaller is the size of the compressed file. Compression speed, on the other hand, is the amount of time required to compress and decompress the image. This value depends on a number of factors, such as the complexity of the algorithm, the efficient of the implementation, and the speed of the processor [13].

\section{G. Motion Estimation Algorithms}

These algorithms assume that a frame has been divided into $\mathrm{M}$ non-overlapping blocks that together cover the entire frame. Moreover, the motion in each block is assumed to be constant, that is, it is assumed that entire block undergoes a translation that can be encoded in the associated motion vector. The problem of block-based ME algorithms is to find the best MV for each block, these algorithms are also called Block Matching Algorithms (BMA) [14].

\section{H. Block Matching Algorithms}

Block Matching Algorithm (BMA) is the most popular technique used for motion estimation in which the current luminance frame is divided into non-overlapped macro blocks (MBs) of size $\mathrm{NxN}$ that are then compared with corresponding macro block (MB) and its adjacent neighbors in the reference frame to create a vector that stipulates the movement of a macro block from one location to another in the reference frame [15], i.e., finding matching macro block of the same size $\mathrm{NxN}$ in the search area in the reference frame.

The position of motion vector has two parts, horizontal and a vertical. These parts can be positive or negative. A positive value means motion was to the right or motion downward while a negative value means motion was to the left or motion upward. This Motion Vector (MV) will be used to predict new frame from the reference which is called motion compensation. The matching measurement is usually determined using one of Block Distortion Measure (BDM) like Mean Absolute Difference (MAD) given by Equation1 or MSE given by Equation2. The macro block with the least cost is considered the matching to the current frame macro block [16]. 


\section{PROPOSED COMPRESSION VIDEO SYSTEM}

$$
\begin{gathered}
M A D=\frac{1}{N^{2}} \sum_{i=0}^{N-1} \sum_{j=0}^{N-1}\left|C_{i j}-R_{i j}\right| \\
M S E=\frac{1}{N^{2}} \sum_{i=0}^{N-1} \sum_{j=0}^{N-1}\left(C_{i j}-R_{i j}\right)^{2}
\end{gathered}
$$

Where:

$\mathrm{N}^{2}$ : Block size $\mathrm{N} x \mathrm{~N}$.

$\mathrm{C}_{\mathrm{ij}}$ : Pixel in position $(\mathrm{i}, \mathrm{j})$ in current block. $\mathrm{R}_{\mathrm{ij}}$ : Pixel in position $(\mathrm{i}, \mathrm{j})$ in reference block.

\section{I. $\quad$ Four Step Search (4SS)}

The 4SS sets a fixed pattern size of $S=2$ for the first step, no matter what the search parameter $p$ value is. Thus, it looks at 9 locations in a $5 \times 5$ window. If the least weight is found at the center of search window the search jumps to the fourth step. If the least weight is at one of the eight locations except the center, then we make it the search origin and move to the second step.

The search window is still maintained as $5 \times 5$ pixels wide. Depending on where the least weight location was, we might end up checking weights at 3 locations or 5 locations. Once again if the least weight location is at the center of the $5 \times 5$ search window we jump to the fourth step or else we move on to the third step. The third is exactly the same as the second step. In the fourth step, the window size is dropped to $3 \times 3$, i.e., $S=1$. The location with the least weight is the best matching macro block and the motion vector is set to point of that location. A sample procedure is shown in Fig, 2 [17].

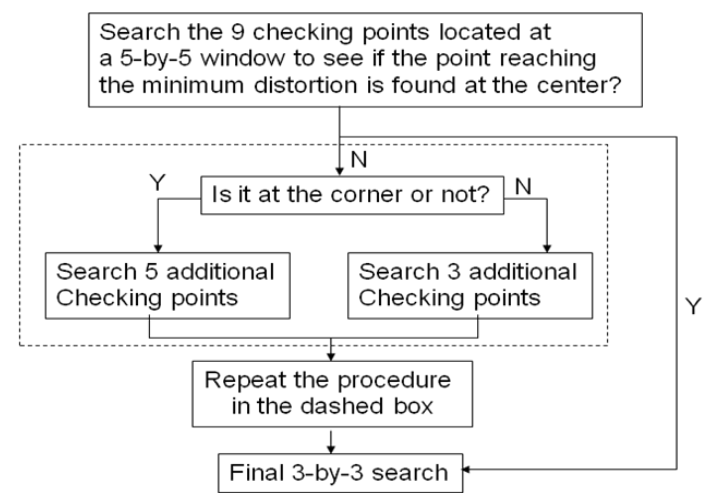

Fig. 2: Flow chart of 4-step search

\section{A. $\quad$ Intra Coded Frame}

The proposed compression system in this system includes three stages, the first stage is the wavelet transform, here we use Haar filter with 1-level. The output from the transform will be different sub bands with different important information. After that, EZW will quantize these sub bands in efficient manner then the output will be stream of zeros and ones, this stream will be compressed by arithmetic encoding, as shown in Fig. 3 .

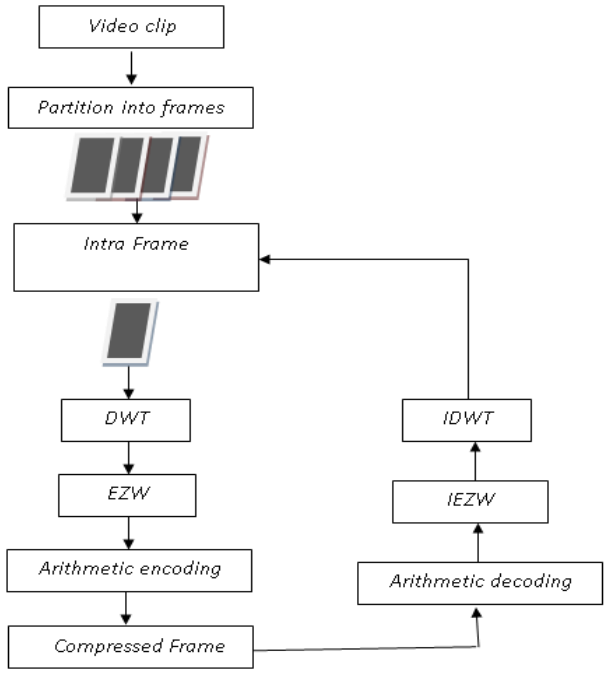

Fig. 3: The flowchart of intra coded frame of clip

\section{B. Inter Coded Frame}

The proposed compression system applies DWT on video frames, then Four Step Search algorithm is used in order to find MV using forward motion estimation. Motion vector was coded by Huffman encoding. On the other hand, the remaining part (the similar blocks of frames) will be compressed as the compression system that was used in intra frame coded, as shown in Fig.4.

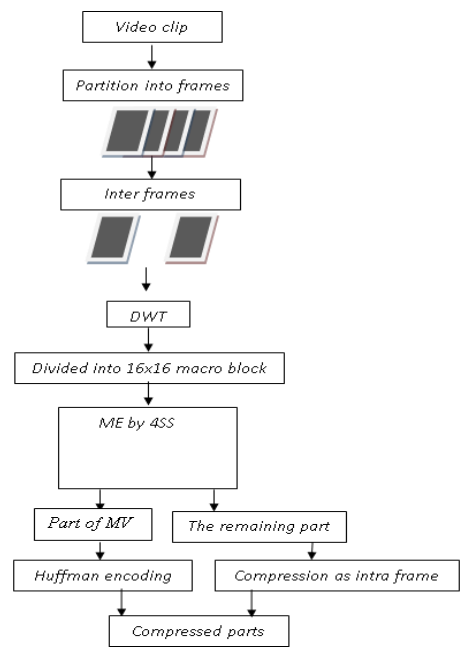

(a): Compression stage

Fig. 4: The flowchart of inter coded frames with in clip. 


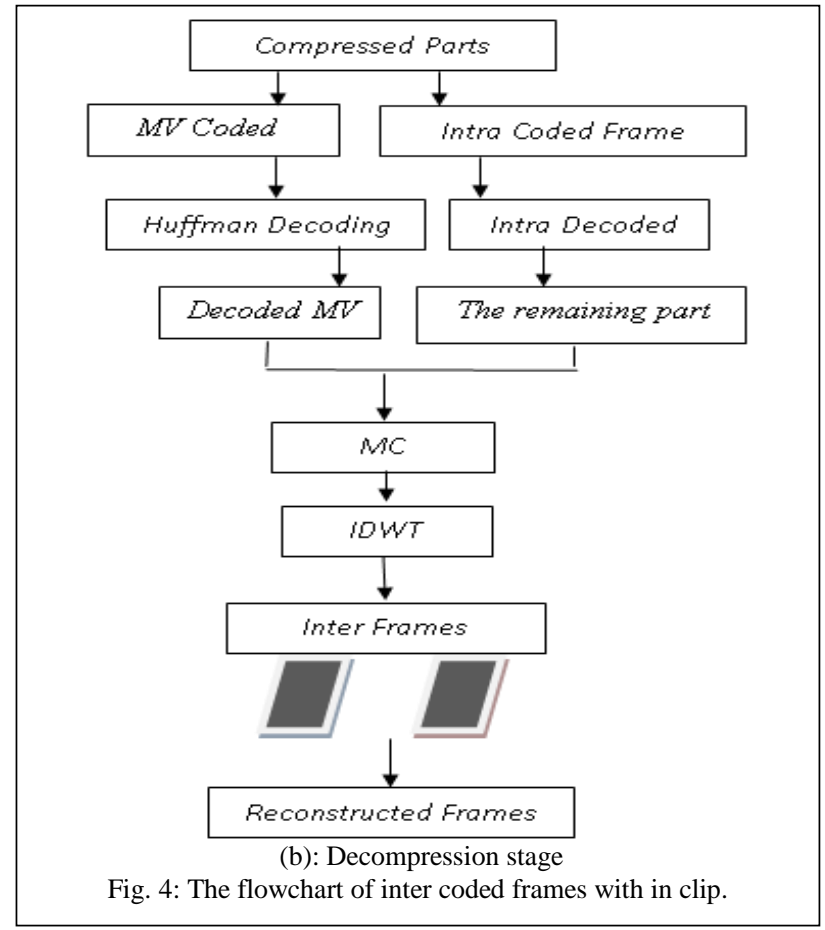

IV. EXPERIMENTAL RESULTS

This section explains the experiments which have been implemented on two video clips. Clip1 and clip 2 as test clips, each one of them is in size of $256 * 256$ and of JPG format. The algorithms are programmed in MATLAB version 7.4.0.287 (R2007a) on a Pentium IV PC (2.4 $\mathrm{GHz}$ ). The first frame in these clips is compressed as intra and the remaining frames as inter through wavelet Haar filter (1 level). This approach was tested by using AVI files format with 5 frames for each clip.

\section{A. Results of Intra Coded Frame}

In this experiment, the first frame of video has been compressed using the first system, namely; intra coded frame. In this system, this frame is compressed as a still image. Two gray scale clips were used in this experiment. Fig. 5 and Fig. 6 show the result of applying this system on these clips. Table1 and Table 2 illustrate the PSNR and CR which are resulted from applying of this system on clip1 and clip2, respectively.
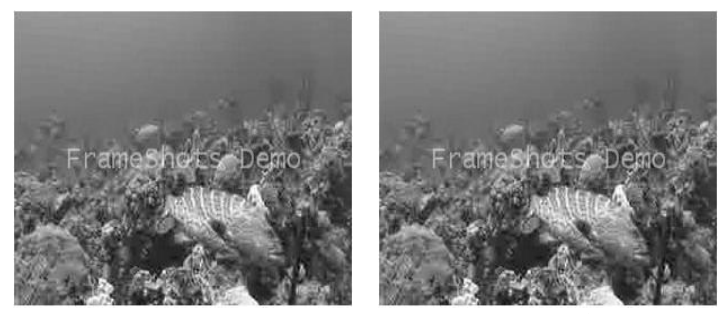

TABLE I

PSNR AND CR OF INTRA CODED SYSTEM ON CLIP

\begin{tabular}{|c|c|c|}
\hline \multirow{2}{*}{ Frame1 } & PSNR $(\mathrm{dB})$ & CR \\
\cline { 2 - 3 } & 61.697 & 0.1447 \\
\hline
\end{tabular}

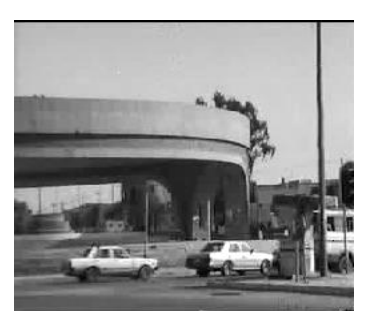

(a)

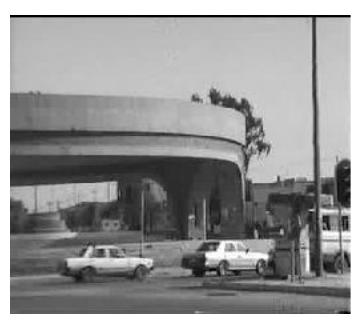

(b)
Fig. 6: Reconstructed frame resulted from applying of the first system on clip2.

Original frame. (b) Reconstructed frame.

TABLE II

PSNR AND CR OF INTRA CODED SYSTEM ON CLIP2.

\begin{tabular}{|c|c|c|}
\hline \multirow{2}{*}{ Frame1 } & PSNR (dB) & CR \\
\cline { 2 - 3 } & 59.389 & 0.1493 \\
\hline
\end{tabular}

\section{B. Results of Inter Coded Frame}

In this experiment, frames starting from the second frame have been compressed using inter coded frame system. In this system, these frames are compressed using DWT followed by motion estimation using 4SS algorithm. Two gray scale clips were used in this experiment. Fig. 7 and Fig. 8 show the result of applying this system on these clips. Table 3 and Table 4 illustrate the PSNR, CR, and processing time which are resulted from applying of this system on clip1 and clip2, respectively.

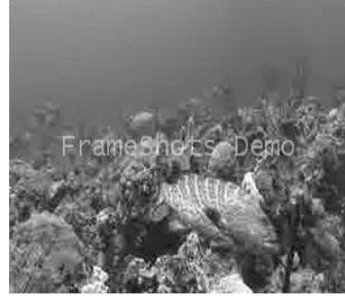

(a)

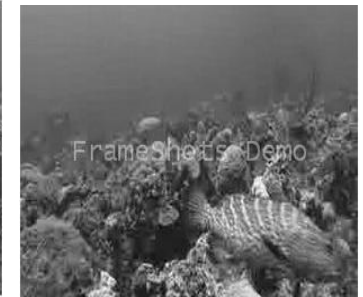

(b)
Fig. 7: Reconstructed frame resulted from applying of the second system on clip1

Original frame. (b) Reconstructed frame.

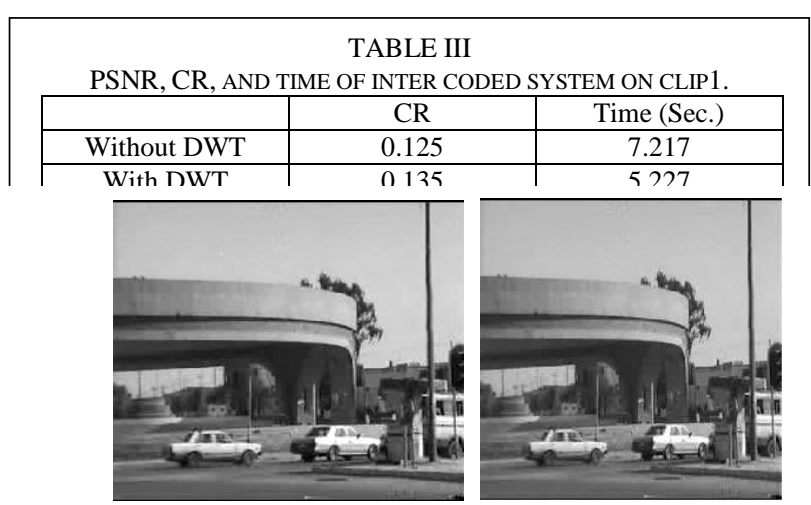

(a)

(b)

Fig. 8: Reconstructed frame resulted from applying of the second system on clip2. 
TABLE III

PSNR, CR, and time of inter coded system on clip1.

\begin{tabular}{|c|c|c|}
\hline & CR & Time (Sec.) \\
\hline Without DWT & 0.135 & 4.6 \\
\hline With DWT & 0.145 & 2.120 \\
\hline
\end{tabular}

\section{DISCUSSION AND CONCLUSIONS}

In this paper, a system for video compression has been proposed. This system based on DWT is used powerful tool in video compression and Four Step Search algorithm as a block matching algorithm to find the motion vector which will be used at the stage of motion compensation to finally estimate the current frame depending on reference frame. We have analyzed the performance of our proposal in terms of both compression ratio and quality (PSNR), and we have visually confirmed these results. We have presented and evaluated several improvements to a compression scheme based on applying the DWT, with the focus on coding video. Use of this system on two clips (one is considered as a standard clip and the another is non standard) has shown good results in terms of PSNR, CR, and processing time. PSNR value in 4SS algorithm is better with DWT proposed approach than this algorithm without PSNR, as shown in Tables 3, and 4 in clip1, and clip 2, respectively. But in $\mathrm{CR}$, the first proposed approach (without DWT) is better than the second proposed approach (with DWT). It is clearly noticed that the use of DWT minimize the processing time, almost, up to $40 \%$ - 50\%. Our coder achieves a good trade-off between compression ratio and quality of the reconstructed video.

\section{REFERENCES}

[1] Sayood K., "Introduction to Data Compression", Morgan Kaufmann Publishers, 2006.

[2] Hassan B. and Malik K. , "Quality-Aware Frame Skipping for MPEG-2 Video Based on Inter Frame Similarity", The Department of Computer Science and Electronics, Malardalen University, Vasteras, Sweden, 2006.

[3] Bjorn B., Sweden, " Image and Video Compression Using Wavelet Transform and Error Robust Transform", M.Sc. Thesis, Stockholm, Sweden KTH Electrical Engineering, September 2005.

[4] Bernabé G., Jose M. Garcia J. M., and Gonzalez J., " A Lossy 3D Wavelet Transform for High-quality Compression of Medical Video", The Journal of System and Software, pp. 526-534, 2009. www.elsevier.com/ locate/ jss.

[5] Nema M., Gupta L., and Trivedi N.R., "Video Compression using SPIHT and SWT Wavelet", International Journal of Electronics and Communication Engineering, Vol. 5, No. 1, pp.1-8, 2012.

[6] Niehsen W., "Fast Full Search Block Matching", IEEE Transactions on Circuits and Systems for Video Technology, pp. 241-247, 2001.

[7] Keinert F., "Wavelets and Multiwavelets", USA, 2004.

[8] Erick S., "Compression of Medical Image Stacks Using Wavelet and Zero-tree Coding", M.Sc. Thesis, Department of Electrical Engineering, Linkoping University, 'Lith-ISY-Ex-3201', 2002.

[9] Saif B., "Wavelet Compression Using Tree and Adaptive Arithmetic Codes", M.Sc Thesis, Baghdad University, College of Science, 2004

[10] "Fast Huffman Code Processing", UCI-ICS Technical Report No. 99-43, Department of Information and Computer Science, University of California, Irvine, October 1999

[11] John M., "Compressed Image File Formats", ACM Press, A Division of The Association of Computing Machinery, Inc. (ACM), 1999.

[12] Salomaon D., "Data Compression, the Complete Reference", Springer-Verlag New York, Inc., USA, 2004

[13] Panrong X., "Image Compression by Wavelet Transform", M.Sc. Thesis, East Tennessee State University, Department of Computer and Information Sciences, 2001.

[14] Djordje M., "Video Compression", University of Edinburgh, 2008. http://homepage.inf.ed.ac.uk/rbf/CVonline/LOCALCOPIES/AVo 506/s0561282.pdf.

[15] Koga T., Iinuma K., Hirano A., Iijima Y. and Ishiguro T., "Motion Compensated Inter Frame Coding for Videoconferencing", Proc. NTC81, Nov. 1981.

[16] Jain J. and Jain A., "Displacement Measurement and Its Applications", IEEE Transactions on Communications, Dec. 1981.

[17] Lai M., and Wing Ch., "A Novel Four Step Search Algorithm for Fast Block Motion Estimation", IEEE Transactions on Circuits And Systems for Video Technology, Vol. 6, No. 3, pp. 313-317, June 1996. 